\title{
To evaluate diagnostic utility of Ultrasonography for palpable thyroid nodule and comparison with FNAC in central India.
}

\author{
${ }^{1}$ *Dr.Piyudeo Mahant, ${ }^{2}$.Dr.Upasana Uniya, ${ }^{3}$ Dr.Narendra Chandel, \\ ${ }^{4}$ Dr.Seema Mahant \\ (Associate Professor-Radiodiagnosis) People's collage of medical science \& Research centre, Bhopal, (M.P.) \\ (Assistant Professor-Pathology) People's collage of medical science \& Research centre, Bhopal, (M.P.) \\ (Assistant Professor-Radiodiagnosis) People's collage of medical science \& Research centre, Bhopal, (M.P.) \\ (Professor-Medicine) People's collage of medical science \& Research centre, Bhopal, (M.P.)
}

\begin{abstract}
:
Objective: Ultrasonographic evaluation of palpable thyroid mass as cystic, solid or mixed, Benign or malignant. To examine the accuracy of Ultrasonographic guided fine needle aspiration cytology (FNAC) in the diagnosis, sensitivity specificity and positive productivity of thyroid nodules.

Methods: This is a prospective study of 100 patients with palpable thyroid nodules who underwent Ultrasonography and finding correlated with diagnostic fine needle aspiration cytology (FNAC) over a 2-year period in central India.

Results: Palpable thyroid nodules are very commonly seen in the population of central India. This study comprises of 100 patients with male female ratio was 1:4.5. Ultrasonographic evaluation done by various investigators, and able to differentiate a mass lesion into cystic, solid or mixed $(100 \%)$. We observed out of 100 lesions 26 as cystic, 28 mixed and 46 as solid lesions which were later proved by FNAC examination and found $100 \%$ accuracy. Among cystic group classification the accuracy was $96 \%$, in solid lesion characterization the diagnostic yield was $87.5 \%$ and for mixed lesion to differentiate benign and malignant lesion the accuracy was $93 \%$.

Conclusion: Ultrasonography is $100 \%$ effective in diagnosing a lesion whether solid, cystic or mixed and help to differentiate a lesion whether malignant or benign. It has added advantage of being safe, reliable, reproducible, cheaper and most important technique without radiation hazard. It also can help in localizing a lesion for biopsy and for guided cyst aspiration. High-resolution thyroid Ultrasonography is a innovative dynamic device for endocrinologists.
\end{abstract}

Key words: Palpable thyroid nodule, FNAC, USG, Sensitivity, Specificity.

\section{Introduction}

Thyroid gland is unique, first to develop and largest endocrine gland with weight about 25 grams superficial in location.(1) Because of superficial location of thyroid glands; high resolution real time grey scale and color Doppler sonography can demonstrate normal thyroid anatomy and pathologic condition with remarkable clarity. As a result, this technique has come to play an increasingly important role in the diagnostic evaluation of the thyroid disease.(2)

Nodular abnormalities of thyroid represent problems of significant. Autopsy studies have demonstrated that the thyroid nodularity is present in approximately $37 \%$ of general population and the incidence of clinically palpable thyroid nodule is just about 4-5\% with overall occurrence of malignancy up to $10-30 \%$.( 3 ) There is a big controversy in the management of palpable thyroid nodule. Few surgeons advised thyroidectomy of all palpable nodule. On the other hand endocrinologist recommend that FNAC be performed as the initial step of evaluation in order to avoid unnecessary surgery. $(4,7)$ Because thyroid nodularity is so common, it would be impracticable to operate every palpable thyroid lesion. So the goal of diagnostic work up now is to select those patients for surgery who have high likelihood of harboring malignancy. Many investigations are used to differentiate between benign and malignant nodule e.g. FNAC, USG, RNS are commonly used. $(2,4,8)$

The present study was undertaken to evaluate the utility of high resolution USG for palpable nodule and comparison with FNAC in differentiating between benign and malignant nodule.

\section{Material and method-}

This is a prospective study was conducted on 100 patients (18 males, 82 females) with an age range of $10-60$ years.

A written "informed consent" was taken from patients before performing Ultrasound and ultrasound guided fine needle aspiration Cytology (US-FNACs). The study was approved by ethical committee. The 
FNACs were collected from patients presented for a thyroid nodule after detailed sonographic evaluation. Evaluation of Patients-The ultrasound machine used was Shimadzu Toshobro Aspire and Shimdzu SD-500 ulttrasound with linear \& convex probe $3.5 \mathrm{MHz}$

Detailed sonographic evaluation particular were noted under following headingsLocation, number Internal content solid, cystic, mixed

Homogenous or heterogenous Parenchymal interface-invisible, echogenic rim Posterior sound transmission-enhancement, shadow, no change.

Lateral edge shadowing present or absent.

Cystic lesions with septations.

Result of the examination was interpreted on basis of these findings and diagnosis was proposed after considering history and physical examination. $(2,5)$

Method of US- FNAC-An experienced radiologist performed an ultrasonographic fine needle aspitaion (USFNAC) with the use $10 \mathrm{ml}$ disposable syringe of (BD Company) with 23-gauge needle by using a perpendicular puncture and without local anesthesia. When the needle tip reached the target nodule, the needle was observed as a small echogenic spot within the nodule on the US monitor. After the needle tip was placed in the appropriate area of the target nodule, sampling was commenced using the "mixed sampling technique". After a sample was obtained, the specimen was mounted immediately onto a glass slide. We obtained four to six slides by performing two or three smears from each nodule. Specimens were fixed with $95 \%$ ethanol and were sent for pathological evaluation. (6)

\section{Results}

The age of patients in this study ranged from 10-60 year. Major affecting group is between 20 to 40 years.

TABLE NO -1 Distribution of subjects by age and sex

\begin{tabular}{|l|l|}
\hline AGE (years) & No. of patients \\
\hline $10-20$ & 12 \\
\hline $20-30$ & 44 \\
\hline $30-40$ & 34 \\
\hline $40-50$ & 8 \\
\hline $50-60$ & 2 \\
\hline MALE/FEMALE & $18 / 82$ \\
\hline TOTAL & 100 \\
\hline
\end{tabular}

TABLE-2 INCIDENCE OF DIFFERENT TYPES OF LESIONS ON SONOGRAPHY

\begin{tabular}{|l|l|}
\hline Type of lesion on sonographic examination & No. of Cases \\
\hline Cystic & 26 \\
\hline Solid & 46 \\
\hline Mixed & 28 \\
\hline Total & 100 \\
\hline
\end{tabular}

TABLE-3 INCIDENCE AND TYPES OF CYSTIC LESIONS ON SONOGRAPHY CORRELATED WITH FNAC

\begin{tabular}{|l|l|l|}
\hline Types of lesions & Sonographic diagnosis & FNAC diagnosis \\
\hline Colloid goiter with cystic degeneration & 18 & 16 \\
\hline Heamorrhagic cyst & 6 & 8 \\
\hline Thyroglosal cyst & 2 & 2 \\
\hline Total & 26 & 26 \\
\hline
\end{tabular}


To evaluate diagnostic utility of Ultrasonography for palpable thyroid nodule and comparison with

TABLE-4 INCIDENCE AND TYPES OF SOLID LESIONS ON SONOGRAPHY CORRELATED WITH FNAC

\begin{tabular}{|c|c|c|}
\hline Types of lesions & Sonographic diagnosis & FNAC diagnosis \\
\hline Benign & 14 & 14 \\
\hline Colloid adenoma & 20 & 14 \\
\hline \multicolumn{3}{|l|}{ Follicular adenoma } \\
\hline Malignant & 4 & 6 \\
\hline Papillary Ca. & 2 & 4 \\
\hline Follicular Ca. & - & 2 \\
\hline Anaplastic Ca. & & \\
\hline Diffused Thyroid Disease & 4 & 2 \\
\hline Graves Disease & 2 & 4 \\
\hline Hashimoto,s Thyroiditis & & \\
\hline Total & 46 & 46 \\
\hline
\end{tabular}

TABLE-5 INCIDENCE AND TYPES OF MIXED LESION ON SONOGRAPHIC EXAMINATION CORRELATED WITH FNAC

\begin{tabular}{|l|l|l|}
\hline TYPE OF LESION & Sonographic Diagnosis & FNAC Diagnosis \\
\hline Benign & 16 & 16 \\
\hline Colloid adenoma with cystic deg. & 4 & 2 \\
\hline Follicular adenoma & 2 & 4 \\
\hline Haemorrhagic cyst & \multicolumn{2}{|l|}{} \\
\hline Malignant & 4 & 4 \\
\hline Papillary Ca. & 2 & 2 \\
\hline Follicular Ca. & 28 & 28 \\
\hline Total & \multicolumn{2}{|}{} \\
\hline
\end{tabular}

TABLE-6 ACCURACY OF SONOGRAPHY IN RELATION TO FNAC IN CHARACTERISING A LESION CYTOLOGICALLY

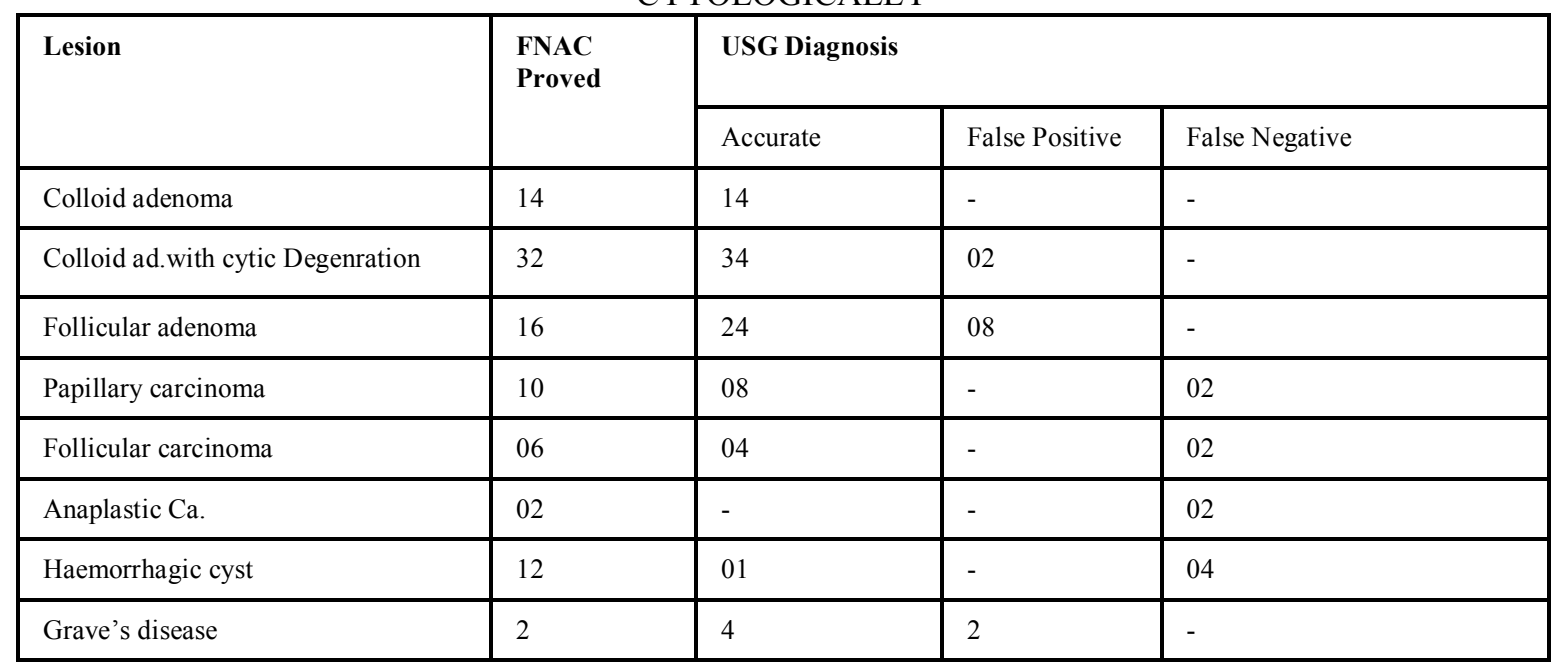

Results - 100 cases of thyroid nodule attending the surgical, ENT, General medicine and endocrine clinic were referred to us with a brief history, physical examination and a provisional clinical diagnosis.

In our study age group range from 6 months to 75 years majority between 20 to 40 years with male female ratio $1: 4.5$

Out of 100 cases, 26 were diagnosed as cystic, 28 were mixed and 46 cases have solid lesions. Diagnostic accuracy was $100 \%$ proved by FNAC examination. 
Solid lesions were categorized in to benign, diffuse thyroid diseases and malignant lesions our diagnostic accuracy was $87.5 \%$.Mixed lesions were categorized into benign and malignant lesions with accuracy rate were 93\%.

So results of our sonographic evaluation of palpable thyroid masses were encouraging giving $100 \%$ accuracy to define categories a lesion as cystic, solid, or mixed lesion and in $90 \%$ cases were giving correct histological diagnosis.

\section{Discussion:}

Ultrasonography is a easily available non-invasive, trouble-free and an truthful modality to evaluate a palpable thyroid nodule. The Revised American Thyroid Association management guidelines for patients with thyroid nodules suggest that, thyroid sonography is mandatory for nodules larger than $10 \mathrm{~mm}$ in diameter because these nodule having the potential to represent a clinically significant cancer.

In previous studies several ultrasonographic characteristics have been studied. These include border characteristics (margins), echogenicity, calcifications, vascularity, size, shape, orientation, and acoustic transmission. A study by Lannuccilli et al assessed the reliability of ultrasonography criteria in labeling a thyroid nodule as benign. Ninety-three percent of the thyroid nodules referred for ultrasonography-guided FNAC were benign and 7\% were malignant. Few other studies shows varies results like-Stacul et al-73\% ,Kim et al $-91 \%$,Kwak et al- $80.6 \%$,Koike et al-91\%,Park et al -92\% ,Horvath et al-88\% specificity for malignancy.

In our study we found $100 \%$ accuracy rate to differentiate cystic, solid and mixed lesion and $87.5 \%$ accuracy rate to distinguish between benign and malignant lesions. So the extensive use of ultrasonography in the evaluation of thyroid nodules has to be established with pliable protocols that minimize costs related to nodule management and maximize the benefits of ultrasonography.

\section{Conclusion:}

Ultrasonography is $100 \%$ effective in diagnosing a lesion whether solid, cystic or mixed and help to differentiate a lesion whether malignant or benign. So it should be used as a valuable adjunct to clinical examination and should be offered to all patients presenting with palpable thyroid nodules. It also has added advantage of being safe, reliable, reproducible, cheaper and most important technique without radiation hazard. It also can help in localizing a lesion for biopsy and for guided cyst aspiration. High-resolution thyroid Ultrasonography is a new dynamic tool for endocrinologists.

\section{Acknowledgement:}

"Feeling can not be felt", expressing the feeling is difficult but when it comes to be expressed in words, it is nearly impossible. But tradition goes on............... So we have to express our gratitude in words to those who contributed and helped us to bring out this work.

First of all we thanks to all our teachers who taught us this subject, than to the patients who made this study easy by their co-operation. At last to all our colleagues and our family members for their motivation.

NO CONFLICT OF INTEREST

\section{Reference:}

[1]. Ilustrated Anatomy of the Head and Neck, Fehrenbach and Herring, Elsevier, 2012, p. 158, ISBN: 9781437724196

[2]. Kerr L. et al: High-resolution thyroid ultrasound: the value of color Doppler ultrasound Quart 1994: 12:21-43.

[3]. Usha Menon V, Sundaram KR, Unnikrishnan AG, Jayakumar RV, Nair V, Kumar H. High prevalence of undetected thyroid disorders in an iodine sufficient adult south Indian population. J Indian Med Assoc 2009;107:72-7.

[4]. Cooper DS, Doherty GM, Haugen BR, Kloos RT, Lee SL, et al. Revised American thyroid association management guidelines for patients with thyroid nodules and differentiated thyroid cancer. Thyroid 2009;19:1167-214.

[5]. Ghassi, D. and Donato, A. (2009) Evaluation of the thyroid nodule. Postgraduate Medical Journal, 85, 190-195. doi:10.1136/pgmi.2008.072140

[6]. Cibas, E.S. and Ali, S.Z. (2009) The Bethesda system for reporting Thyroid Cytopathology. American Journal of Clinical Pathology, 132, 658-665. doi:10.1309/AJCPPHLWMI3JV4LA

[7]. Kovacevic, D.O. and Skurla, M.S. (2007) Sonographic diagnosis of thyroid nodules: Correlation with the results of sonographically guided fine-needle aspiration biopsy. Journal of Clinical Ultrasound, 35, 63-67. doi:10.1002/jcu.20287

[8]. Frates, M.C., Benson, C.B., Charboneau, J.W., Cibas, E.S., Clark, O.H., Coleman, B.G., et al. (2005) Management of thyroid nodules detected at US: Society of radiologists in ultrasound consensus conference statement. Radiology, 237, 794-800. doi:10.1148/radiol.2373050220

[9]. Lin JD, Chao TC, Huang BY, Chen ST, Chang HY \& Hsueh C. Thyroid cancer in the thyroid nodules evaluated by ultrasonography and fine-needle aspiration cytology. Thyroid $200515708-717$. 